\title{
Magnetic reconnection between an emerging active region and the quiet Sun
}

\author{
Bin Zhang ${ }^{1,2}$, Jun Zhang ${ }^{1}$, Shuhong Yang ${ }^{1}$, Ting $\mathbf{L i}^{1}$, Yuzong Zhang ${ }^{1}$ \\ and Leping $\mathbf{L i}^{1}$ \\ ${ }^{1}$ Key Laboratory of Solar Activity, National Astronomical Observatories, \\ Chinese Academy of Sciences, Beijing 100012, China \\ email: zjun@nao.cas.cn \\ ${ }^{2}$ The Pilot College of Beijing University of Technology, Beijing 101101, China
}

\begin{abstract}
Using the Solar Dynamics Observatory observations, we study the evolution of an emerging active region (EAR) and its reconnection with the quiet Sun. The EAR continuously interacts with the surrounding quiet region, and dark ribbons at the boundary of the EAR and the quiet Sun are observed. The extreme-ultraviolet observations show that the regions swept by the dark ribbons are brightened and the temperature increases. These results reveal that there exists an uninterrupted magnetic reconnection between the EAR and the quiet region and the released energy heats the corona of the quiet Sun. The dark ribbons are suggested to correspond to the interface of the reconnected fields and the undisturbed ones. The dark ribbon propagates outward, and this phenomenon is considered as a dark wave.
\end{abstract}

Keywords. Sun: activity, Sun: corona, Sun: evolution

\section{Introduction}

Magnetic reconnection which is a rearrangement of field topology is a fundamental physical process in conductive plasma. During the magnetic reconnection, magnetic energy is converted to the kinetic and thermal energy, which is responsible for solar flares and many types of outbursts (e.g., Yang et al. 2011; Zhang et al. 2013; Yang et al. 2014; Yang et al. 2015). Free magnetic energy rapidly increases during flux emergence, and the coronal field develops a tangential discontinuity at the interface between the preexisting and newly emerged flux systems. On one side of the discontinuity, field lines have footpoints wholly within the preexisting flux system, and on the other side the lines wholly within the newly emerged system. When reconnection occurs, field lines from the two sides exchange footpoints, and new field lines connecting new to old flux are created. These new field lines retract and compress, adding kinetic and thermal energy to the plasma (Reeves et al. 2008; Guidoni \& Longcope 2010).

Rapid reconnection usually triggers a flare, and the observational features are flare ribbons, which are observed in either $\mathrm{H} \alpha$ or $1600 \AA$ data (Fletcher \& Hudson 2001; Qiu 2009). In a flare, the flux transferring and resulting energy release typically take about $30 \sim 60 \mathrm{~min}$. If the energy released is insufficient, then the rate of reconnection cannot be determined from the flare ribbon expansion. A number of observational studies have suggested that reconnection is not directly associated with flares, especially in the quiet Sun (Schrijver et al. 1997).

Recently, a quantitative measurement of the amount of the rate of reconnection $\left(\sim 10^{16}\right.$ $\mathrm{Mx} \mathrm{s}^{-1}$ ) during quiescent reconnection between an emerging flux bundle and the surrounding preexisting field has been reported (Tarr et al. 2014). There exists a basic question that at which condition reconnection occurs very rapidly, and at which 


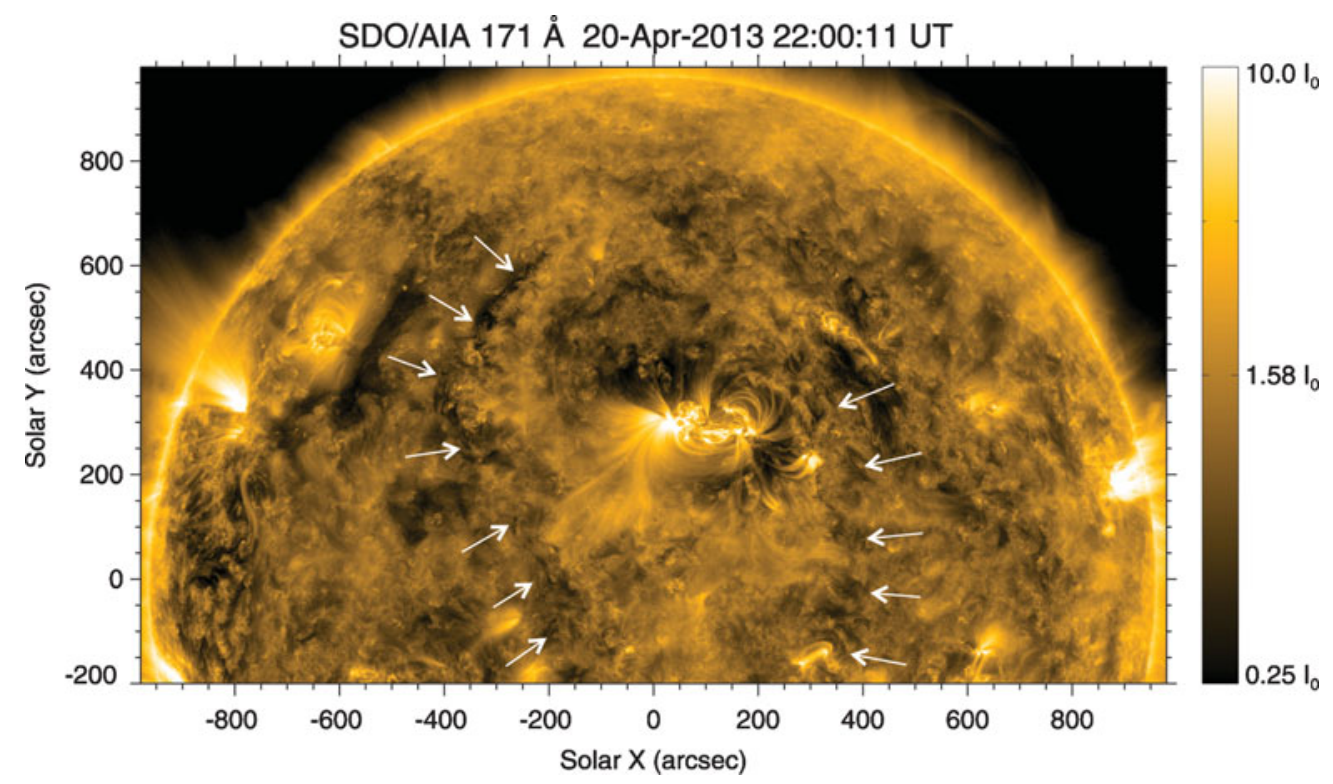

Figure 1. $171 \AA$ image showing the dark ribbons (denoted by white arrows) which propagated away from the AR 11922, when the AR developed.

condition the reconnection is more slowly. This fundamental question concerning magnetic reconnection will need to be addressed in details.

When we check the Atmospheric Imaging Assembly (AIA; Lemen et al. 2012) movies day by day from the Solar Dynamics Observatory (SDO; Pesnell et al. 2012) website, we find a new phenomenon, i.e., emerging active regions (EARs) on the solar disk are always accompanied by extreme-ultraviolet (EUV) dark ribbons which propagate outward from the EARs (Zhang et al. 2015). In the present paper, we study the interaction between an EAR and the quiet Sun, and the accompanied dark ribbons.

\section{Observations and results}

The SDO/AIA detects uninterruptedly the full disk of the Sun in 10 wavelengths at a 12 -second cadence and a $0^{\prime \prime} .6$ pixel $^{-1}$ sampling. The data reflect different temperatures (from $\sim 5000 \mathrm{~K}$ to $\sim 20 \mathrm{MK}$ ) from the photosphere to the corona. The Helioseismic and Magnetic Imager (HMI; Scherrer et al. 2012) on board the SDO measures the Doppler velocity, line-of-sight (LOS) magnetic field, and vector magnetic field at the photosphere of the Sun. The data cover the full disk of the Sun with a spatial sampling of $0^{\prime \prime} .5$ pixel $^{-1}$. The full disk LOS magnetograms are taken at a cadence of 45 seconds. The data used in this study were obtained from April 17 to April 23 in 2013.

On 2013 April 17, a dipole appeared at N13E35. Its magnetic flux grew evidently, and then the dipolar EAR was named as AR 11726 (see Fig. 1) on April 19. Emerging dimming (Zhang et al. 2012) appeared at 18:15 UT on 2013 April 18, and lasted almost 50 hours. This EAR continuously (last almost a hundred hours) interacted with the surrounding quiet Sun, resulting in dark ribbons (marked by the white arrows in Fig. 1) which first appeared around 21:16 UT on 2013 April 19 at the east interface of the EAR and the quiet Sun.

The area of the AR reached $1.1 \times 10^{4} \mathrm{Mm}^{2}$, the total magnetic flux of the AR was $6.5 \times 10^{21} \mathrm{Mx}$, and the net magnetic flux was positive $\left(6.6 \times 10^{20} \mathrm{Mx}\right)(\mathrm{Fig} .2 \mathrm{~A})$. As the 

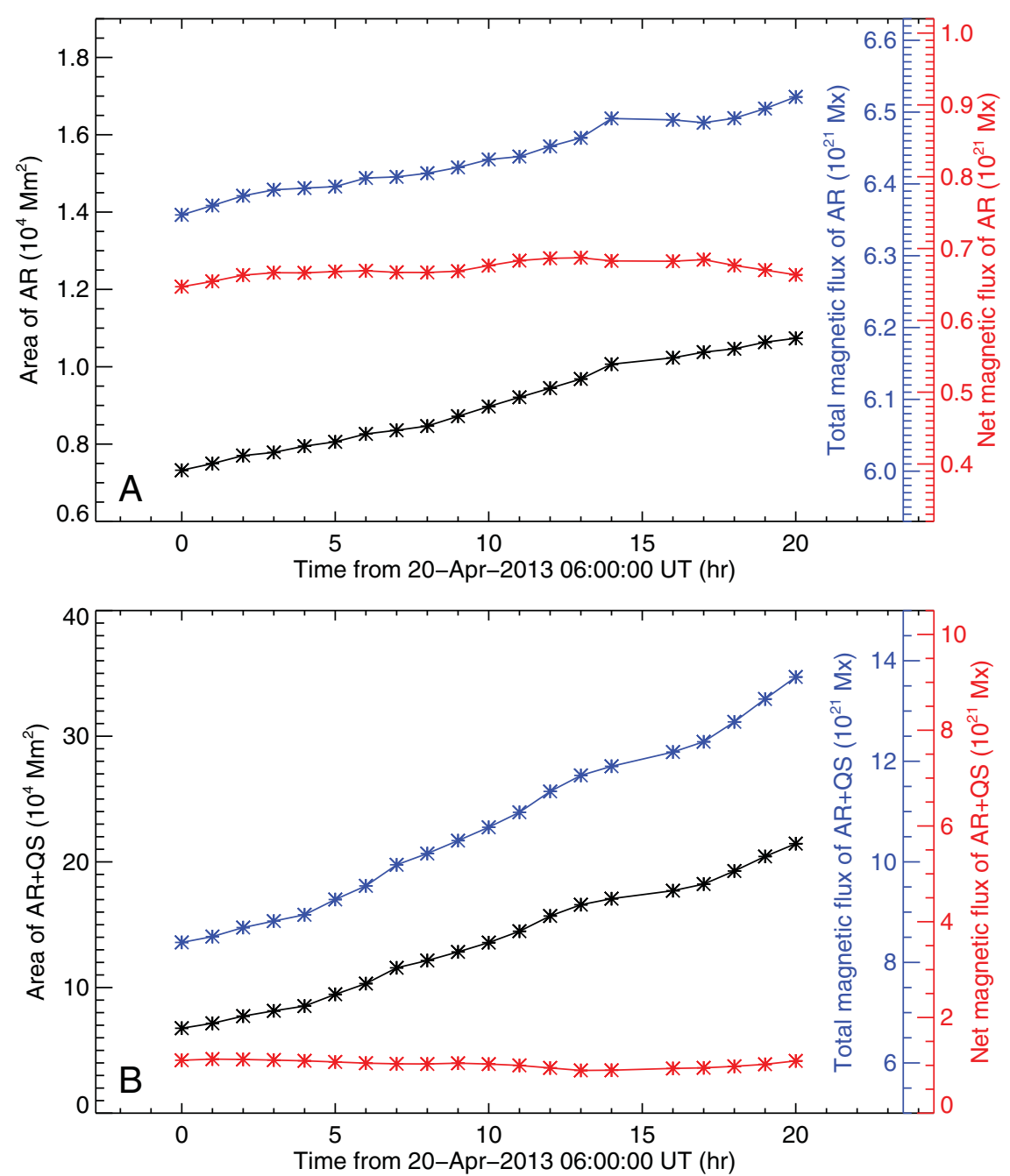

Figure 2. (a) Temporal evolution of active region area, total magnetic flux and net flux. (b) Temporal evolution of area, total flux and net flux inside the region swept by the dark ribbons.

AR developed, the dark ribbons propagated outward, and the area encircled by the ribbons enlarged ((Fig. 2B)). At 02:00 UT on 2013 April 21, the area was $2.0 \times 10^{5} \mathrm{Mm}^{2}$, about twenty times larger than that of the AR.

During the EAR developing, the region previously swept by the dark ribbon brightened up (Figs. 3A-C). At 13:30 UT on 2013 April 20, the dark ribbon (marked by the arrows in Fig. 3A) was at the west boundary of the ringlike region (a partial coronal hole, outlined by closed red dashed curve). Almost 33 hours later (Fig. 3C), the ringlike region had been swept by the dark ribbon. The EUV radiation became stronger in this region, and the partial coronal hole disappeared. The brightness increased by about $27 \%$, although the magnetic flux did not change evidently during this period (Fig. 3E). The mean temperature in the closed curve region swept by the dark ribbon increased by $23 \%$ (from $1.3 \mathrm{MK}$ to 1.6 MK). Emission maps show that the density also increased evidently, e.g., the mean density in the closed curve region increased by $47 \%$ (from $1.7 \times 10^{26} \mathrm{~cm}^{-5}$ to $2.5 \times 10^{26}$ $\mathrm{cm}^{-5}$ ). It should be noted that the temperatures of the active region, the neighboring 


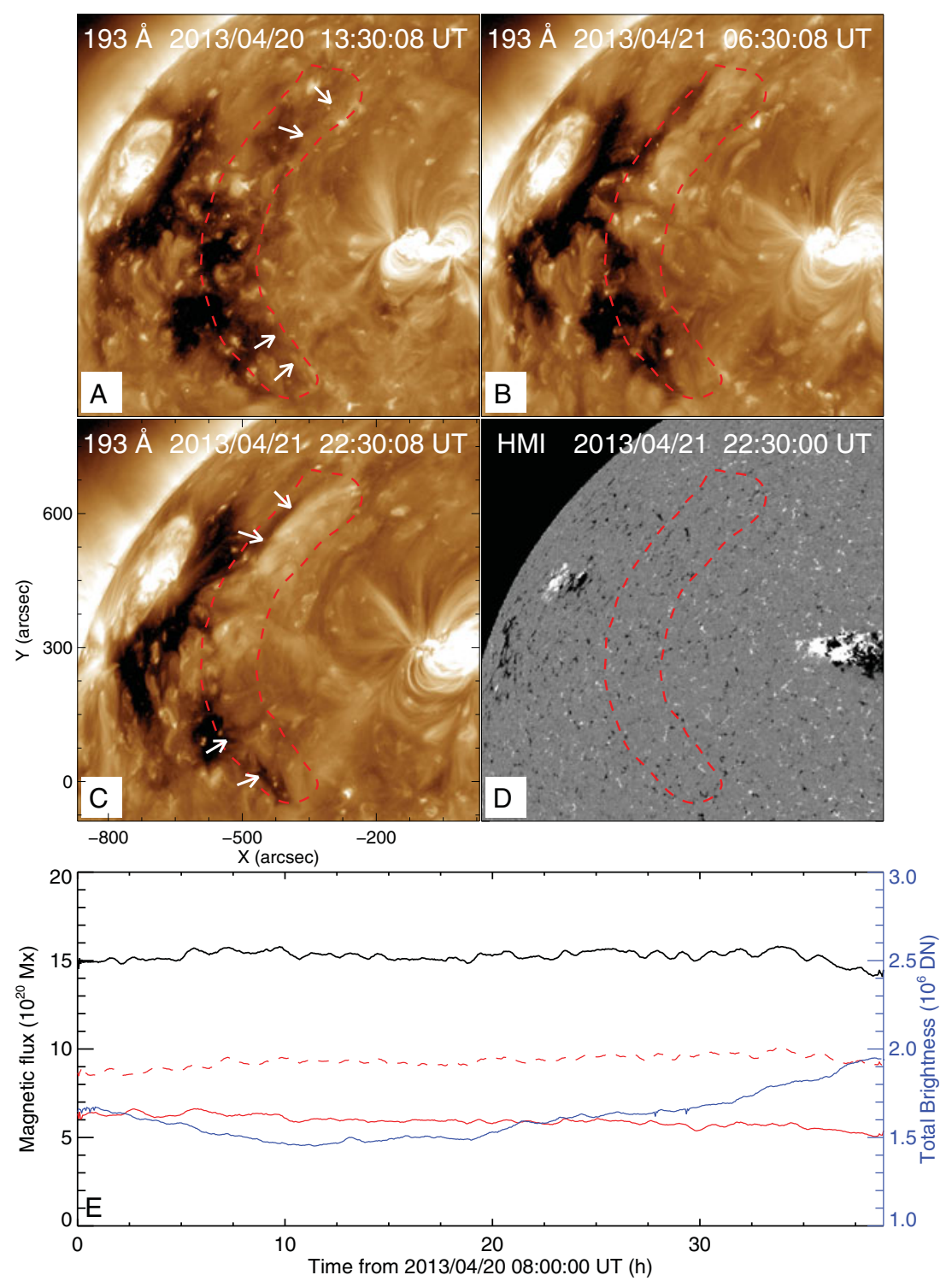

Figure 3. Panels (a) - (c): three AIA $193 \AA$ images showing the evolution of coronal hole brightness when the dark ribbon swept across the hole. Arrows in panels (a) and (c) mark the ribbon. The region outlined by the dash line is swept by the ribbon. Panel (d): the corresponding HMI magnetogram. Bottom: temporal evolution of the positive (red solid curve), negative (red dash curve) and total (black solid curve) flux in the region. The blue solid curve shows the evolution of the EUV 193 A brightness.

quiet region, and the remote coronal hole are obtained with the differential emission measure (DEM) analysis method which is based on the "xrt_dem_iterative2.pro" in the Solar Software package (Cheng et al. 2012). In this method, a DEM profile is guessed at first and then folded through the response of each wavelength, and thus produce predicted fluxes. To make sure the predicted fluxes are very close to the observed fluxes, we iterate this process using the Levenberg-Marguardt least-square minimization. It should be mentioned that we also interpolate the DEM profile using $\mathrm{N}-1$ spline functions, 

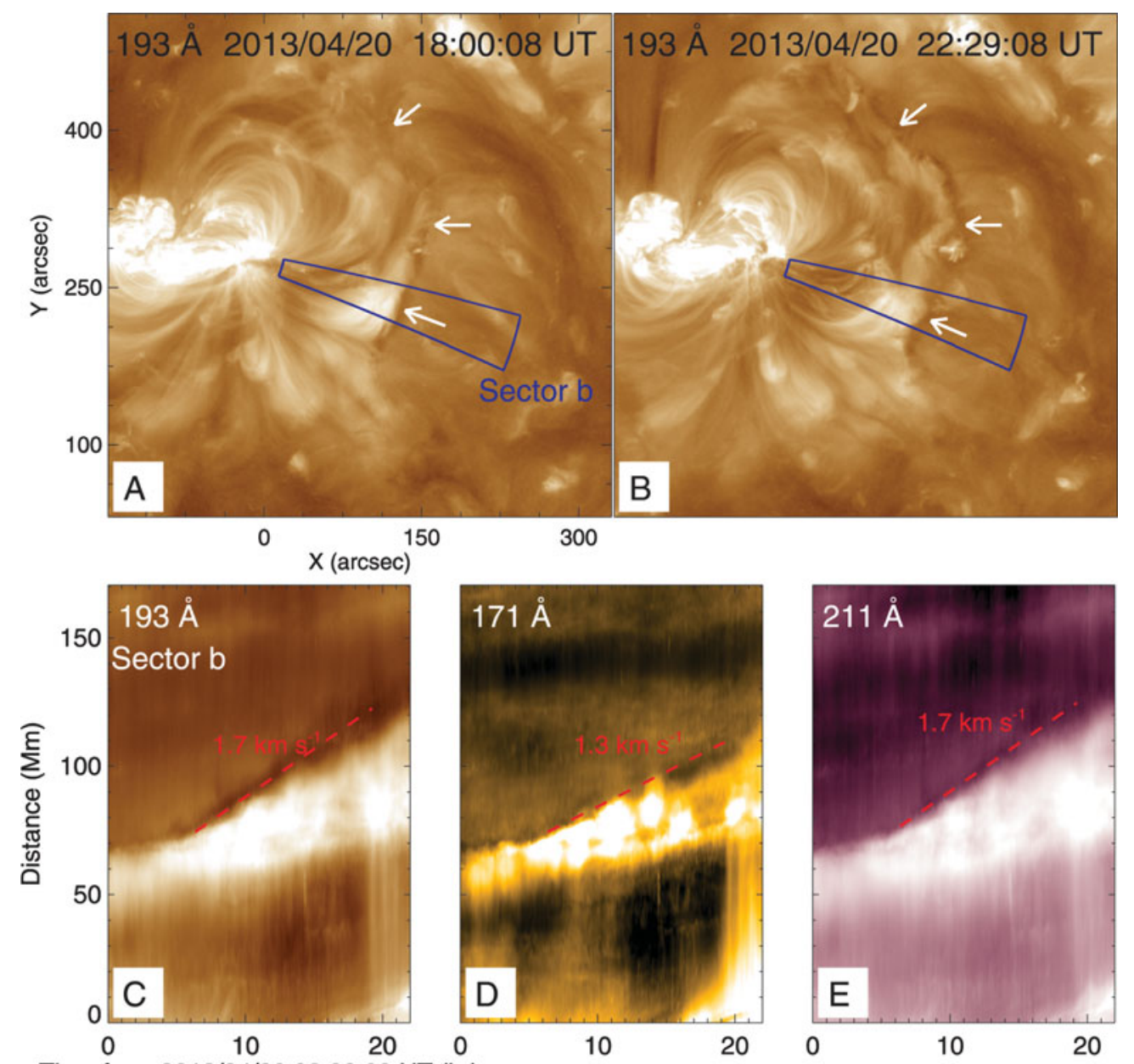

Time from 2013/04/20 08:00:00 UT (hr)

Figure 4. Panels (a) and (b): two AIA $193 \AA$ images showing the propagation of another dark ribbon (denoted by arrows). Panels (c) - (e): three stack plots along sector "B" (see panels (a) and (b)) in 193, 171, and $211 \AA$ showing the propagating speed of the dark ribbon.

representing the freedom degrees for $\mathrm{N}$ different wavelength observations. In this study it is based on the AIA observations in four lines (171 $\AA, 193 \AA, 211 \AA$, and $335 \AA$ ), considering the lower temperature and density of the quiet region and coronal hole. The emission measure (EM) maps of the active region, quiet region, and coronal hole are also obtained with the DEM analysis method.

The dark ribbon can not be detected near 19:30 UT on 2013 April 23, when it met the boundary of another active region AR 11727. On the west of the EAR, another dark ribbon (denoted by arrows in Figs. 4A-B) propagated westward with speeds from $1.3 \mathrm{~km}$ $\mathrm{s}^{-1}$ to $1.7 \mathrm{~km} \mathrm{~s}^{-1}$ (Figs. 4C-E). At the late phase of the EAR development, almost all the northern hemisphere of the visible disk was affected by the dark ribbon.

\section{Conclusions and Discussion}

With the SDO observations, we study the evolution of an EAR and its interaction with the quiet Sun. The EAR reconnects with the surrounding quiet Sun continuously, 
and dark ribbons at the boundary of the EAR and the quiet Sun are observed. The EUV observations show that the regions swept by the dark ribbons are brightened and the temperature increases. These results reveal that there exists an uninterrupted magnetic reconnection between the EAR and the quiet Sun and the released energy heats the corona of the quiet Sun. The dark ribbons are suggested to correspond to the interface of the reconnected fields and the undisturbed fields. Their propagating speed reflects the reconnection rate in some sense.

In this work, the magnetic reconnections between the EARs and the surrounding quiet Sun change the configuration of the quiet Sun fields systematically. The magnetic reconnections comb the quiet Sun fields, e.g., making the formerly arbitrary distributing magnetic fields regular distributing, thus the magnetic fields are arranged along specifically directions. Since both the plasma and the magnetic fields are frozen together, during the combing (magnetic reconnection) process, an instantaneous void region (where the magnetic strength is smaller and the plasma density is lower) appears between the combed fields and the uncombed ones, and EUV observations display that there exists a dark ribbon. As the reconnection develops, the peripheral fields reconnect in turn, subsequently new instantaneous void regions appear in turn also. The observations show that the dark ribbon propagates outward, and this phenomenon is considered as a dark wave.

\section{Acknowledgements}

This work is supported by the National Natural Science Foundations of China (11533008, 11203037, 11221063, 11303050, and 11303049), the Youth Innovation Promotion Association of CAS (2014043), and the Strategic Priority Research Program-The Emergence of Cosmological Structures of the Chinese Academy of Sciences (No. XDB09000000).

\section{References}

Cheng, X., Zhang, J., Saar, S. H., \& Ding, M. D. 2012, ApJ, 761, 62

Fletcher, L. \& Hudson, H. 2001, Solar Phys., 204, 69

Guidoni, S. E. \& Longcope, D. W. 2010, ApJ, 718, 1476

Lemen, J. R., Title, A. M., Akin, D. J., et al. 2012, Solar Phys., 275, 17

Pesnell, W. D., Thompson, B. J., \& Chamberlin, P. C. 2012, Solar Phys., 275, 3

Qiu, J. 2009, ApJ, 692, 1110

Reeves, K. K., Seaton, D. B., \& Forbes, T. G. 2008, ApJ, 675, 868

Scherrer, P. H., Schou, J., Bush, R. I., et al. 2012, Solar Phys., 275, 207

Schrijver, C. J., Title, A. M., van Ballegooijen, A. A., Hagenaar, H. J., \& Shine, R. A. 1997, ApJ, 487, 424

Tarr, L. A., Longcope, D. W., McKenzie, D. E., \& Yoshimura, K. 2014, Solar Phys., 289, 3331

Yang, S., Zhang, J., Li, T., \& Liu, Y. 2011, ApJL, 732, L7

Yang, S., Zhang, J., \& Xiang, Y. 2014, ApJL, 793, L28

Yang, S., Zhang, J., \& Xiang, Y. 2015, ApJL, 798, L11

Zhang, J., Yang, S., Liu, Y., \& Sun, X. 2012, ApJL, 760, L29

Zhang, J., Yang, S., Li, T., et al. 2013, ApJ, 776, 57

Zhang, J., Zhang, B., Li, T., et al. 2015, ApJL, 799, L27 